Bull. Egypt. Soc. Physiol. Sci. Vol. (41) Issue (1), 121-139

\author{
Bull. of Egyp. Soc. Physiol. Sci. \\ (Official Journal of Egyptian Society for Physiological Sciences) \\ (pISSN: 1110-0842; eISSN: 2356-9514)
}

\title{
Exercise alleviates osteoporotic changes in glucocorticoids- induced osteoporosis in a rat model: a possible role of endogenous irisin
}

\section{Manar Shabanah, Mohamed Adel ${ }^{*}$, Shereen M. Samir ${ }^{1}$, Gad Elmawla A. Gad and Hanaa G. Elserougy}

\author{
Department of Medical Physiology, Faculty of Medicine, Mansoura University, Egypt
}

\begin{abstract}
Submit Date: May 5, 2020

Accept Date : August 10, 2020

Available Online: Jan 1, 2021

Keywords

- $\quad$ Iirisin

- Osteoporosis

- Exercise

Irisin, the newly identified myokine, is produced by skeletal muscle in response to exercise. The aim of our work is to study the effect of exercise on irisin plasma level and identify the correlation between irisin level in blood and change in bone turnover in glucocorticoids- induced osteoporosis. Forty eight Sprague-Dawley rats were divided equally into four groups: control group, containing sedentary rats; exercise group, these rats were subjected to a swimming exercise protocol ( 5 days per week for 8 weeks) in a gradual manner; osteoporotic group, rats received methylprednisolone, intramuscular, $(0.2 \mathrm{mg} / \mathrm{kg}$ three times/ week) for 5 weeks; and exercise - osteoporotic group, exercise training combined with osteoporosis: these rats were subjected to swimming exercise in the same previous protocol and after 3 weeks they received methylprednisolone. Exercise group showed a significant increase in cortical bone thickness and trabecular bone area \%. Exercise increased serum irisin level and decreased serum C-terminal telopeptide of type I collagen (CTX) level in exercise group. Also, cortical bone thickness and trabecular bone area \% increased relative to total bone area in exerciseosteoporotic group. The results revealed a positive correlation between serum irisin level and bone mineral content, bone mineral density, microstructure of bone and osteocalcin expression. Also, there is a negative correlation between serum irisin level and CTX level. So, irisin may be the link between the beneficial effects of exercise and the protection against osteoporosis. So, recombinant irisin may be a crucial osteoprotective intervention for those patients whose plasma irisin level is reduced.
\end{abstract}

Corresponding author: Mohamed Adel, madel7744@yahoo.com or madel@mans.edu.eg; Tel.: +20-1222218973, Department of Medical Physiology, Faculty of Medicine, Mansoura University, Egypt 


\section{Introduction}

Osteoporosis is the most common bone disease in humans, representing a major public health problem (1). It is characterized by low bone mass, deterioration of bone tissue and disruption of bone architecture, compromised bone strength and an increase in the risk of fracture which occurring spontaneously or following minor trauma (2). Papaioannou et al. (3) reported that osteoporosis fractures cause excess mortality, morbidity, chronic pain and economic costs. According to the WHO diagnostic classification (2004), osteoporosis is defined by bone mineral density (BMD) at the hip or lumbar spine that is less than or equal to 2.5 standard deviations below the mean BMD of a young-adult reference population. So, any disorder that alters bone formation and enhances bone resorption will decrease BMD and triggers development of osteoporosis. Osteoporosis is classified into primary and secondary types. Primary osteoporosis is regarded as bone loss associated with the aging process (4). It is diagnosed when no disease causing low bone mineral density other than osteoporosis and bone mass is about $70 \%-80 \%$ of young adult male (4). But, secondary osteoporosis is bone loss that results from many other factors such as nutritional deficiencies that adversely impact bone metabolism (5). Certain medications may also increase the rate of secondary osteoporosis including steroids, some antiseizure medications, chemotherapy, proton pump inhibitors and selective serotonin reuptake inhibitors (6). Some chronic diseases may lead to secondary osteoporosis as chronic obstructive pulmonary disease and renal insufficiency or renal failure (7).
Although, it is well known that there is a clear link between physical activity and bone acquisition and maintenance (8), the question of how muscle function regulates bone mass is not fully understood yet.

Cosman et al. (9) found that physical exercise is one of the most important non pharmacological prevention and therapeutic protocols for osteoporosis. Electrical stimulation of muscle in rats with experimental spinal cord injury has been shown to attenuate bone resorption and osteoclastogenesis, providing direct evidence for muscle-bone communication (10). Recently, it has been reported that during or immediately after physical activity, skeletal muscle releases several hormones into circulation. These hormones, named myokines, include IL6, IL5 and most recently, irisin (11).

Irisin, the newly identified myokine, is produced by skeletal muscle in response to exercise (12). It is 112 amino acid residues' glycosylated protein hormone 13. Boström et al. (11) reported that certain receptors in muscle named peroxisome proliferator-activated receptor gamma coactivator 1-alpha (PPARy, also known as PGC1- $\alpha$ ) during exercise has been shown to stimulate the production of the membrane protein fibronectin type III domain containing protein 5 (Fndc5). The latter is subsequently cleaved to, and released as, irisin. It is considered that irisin mediates the benefits of exercise by promoting the browning of white adipose tissue, which results in enhanced thermogenesis and increased energy expenditure (14). The induction of browning in white adipose tissue could be an effective strategy to increase lipid metabolism and improve obesity and type 2 diabetes (15). The relationship between 
irisin and osteoblast differentiation is still a matter of controversy; in vitro studies have recorded a positive (16), negative (17), and even no correlation (18). Moreover, available data on whether the circulating irisin level is associated with osteoporotic fractures or not are limited.' Instead of 'But to date, there have been reports of the positive (16), negative (17) and no correlations between irisin and osteoblast differentiation in vitro (18) and if circulating irisin level was associated with osteoporotic fractures or not (19).

So, the aim of our work is to: (i) study the effect of exercise on irisin plasma level in rats (ii) identify the correlation between irisin level in blood and change in bone turnover in corticosteroid induced osteoporosis.

\section{Materials and methods}

\subsection{Experimental animals}

Forty eight male Sprague Dawley rats, weighing 350-400gm, and aged 8-12 weeks old were used in this study. The animals were purchased and housed in the Medical Experimental Research Centre (MERC). They were housed in a controlled environment that was maintained under a 12 hour light/dark cycle and a temperature of $25^{\circ} \mathrm{C}\left( \pm 3^{\circ} \mathrm{C}\right)$. The rats were allowed free access to a standard diet and water. This research was approved by the Medical Research Ethics Committee of Mansoura University.

\subsection{Experimental design}

Forty eight Sprague-Dawley rats were divided equally into four groups: control group (Control), containing sedentary rats; exercise group, these rats were subjected to a swimming exercise protocol (5 days per week for 8 weeks) in a gradual manner (17); osteoporotic group, rats received methylprednisolone, intramuscular, $(0.2$ $\mathrm{mg} / \mathrm{kg}$ three times/ week) for 5 weeks; and exercise - osteoporotic group, exercise training combined with osteoporosis: these rats were subjected to swimming exercise in the same previous protocol and after 3 weeks they received methylprednisolone in the same dose as previously mentioned.. At the end of eighth week, rats of all groups were anesthetized with intraperitoneal thiopental (30 mg/kg) (18), then blood collected from the heart by a syringe. This blood was put in a clean centrifuge tube. Then it was left to coagulate and then centrifuged at $3000 \mathrm{rpm}$ for 30 minutes to separate the serum. This serum was stored at $-20^{\circ} \mathrm{C}$ for biochemical analysis.

\subsection{Swimming exercise protocol}

Animal were made to swim in a cylindrical glass tank (80 cm diameter, $100 \mathrm{~cm}$ height, $40 \mathrm{~cm}$ depth) filled with water maintained at $35^{\circ} \mathrm{C}$. Exercise was done at the same time daily (9:00-11:00 a.m.) for the calculated period. Rats were acclimatized to swimming one week before the experiment. Initially rats swam for 10 minutes during the first week, with increment of additional 5 minutes daily until swimming period of 35 minutes was attained. Subsequently, a daily swimming period of 35minutes, 5 times /week, was maintained for 8 weeks (17). At the end of each exercise session, animals were dried and kept in a warm environment.

\subsection{Determination of serum calcium level}

Calcium ions react with o-cresolphtaliencomplex in an alkaline medium to form a purple coloured complex (17). We utilized kits purchased from Human Gesellschaft für Biochemica company, Germany. The absorbance of this complex is proportional to calcium concentration in the samples, the absorbance of these samples 
were read using spectrophotometer adjusted at 450 nm.

\subsection{Determination of serum irisin level}

Irisin level was determined by using rat irisin ELISA kit purchased from Kono Biotech Company. Ltd, Zhejiang, China. Purified rat irisin antibody was used to coat microtiter plate wells to make a solid-phase antibody, then irisin was added to wells, combined antibody which with HRP labeled goat anti-rat become antibody-antigenenzyme-antibody complex. After washing completely, 2, 2, 6, 6-Tetramethylpiperidine (TMP) substrate was added to obtain blue color. The reaction was terminated by the addition of sulphuric acid solution and the color change is measured spectrophotometrically at a wavelength of $450 \mathrm{~nm}$. The concentration of rat irisin in the sample was then determined by comparing the optical density of the sample to the standard curve.

\subsection{Determination of serum C-terminal telopeptide of type I collagen (CTX) level}

CTX level was determined by using rat ELISA kit purchased from Bio -Rad Inc., Hercules, CA, USA. This assay employs the competitive inhibition enzyme immunoassay technique. The microtiter plate provided in this kit was pre-coated with CRISP3. Samples were added to the appropriate microtiter plate wells with Horseradish Peroxidase (HRP) conjugated antibody preparation specific for CRISP3. The competitive inhibition reaction was launched between with pre-coated CRISP3 and CRISP3 in samples. A substrate solution was added and the color established is opposite to the amount of CRISP3 in the samples. The intensity of the color is measured (6).

2.7. Dual energy X-ray absorptiometry (DEXA) on left tibia
Bone mass was measured using a dual X-ray absorptiometry device (DXA-Hologic model 4500A, Waltham, MA, USA) at baseline and at the end of the experiment. Animals were anesthetized with intraperitoneal xylazine $(20 \mathrm{mg} / \mathrm{kg})$ and ketamine $(40 \mathrm{mg} / \mathrm{kg})$. They were placed in a prone position for bone mass measurements. Bone mineral density (BMD, $\mathrm{g} / \mathrm{cm}^{2}$ ) and bone mineral content (BMC, g), were determined using specific small animal software (Version 610-0691 for QDR $\mathrm{XP})$.

\subsection{Histopathological examination of right tibia}

The rat was stretched on a wax platform and fixed with metal needles from the left hindlimb to the right forelimb. The skin was gently raised and snipped away from the muscle. The skin was peeled from the muscles, using scissors and a probe to tease away muscles that stick to the skin. Carefully, the biceps femoris and gastrocnemius were teased away to expose the leg bones. The distal end of the tibia was isolated and cut within the ankle joint cavity. The muscles were retracted over the knee joint; the femur was held by a pair of scissors at the proximal end of the knee joint space and gently twisted toward the reversed genuflex of the knee joint. At this point, the entire proximal end of the tibia (Knee joint end) was popped out and completely isolated then clean from any remaining muscle. Rat tibias were dissected out, fixed in $10 \%$ neutral buffered formaldehyde for 2 days and decalcified in EDTA solution for 4 weeks (11). The decalcified specimens were dehydrated and processed to form paraffin blocks. Serial transverse and longitudinal sections ( $5 \mu \mathrm{m}$ thick) from the diaphysis and metaphysis of tibia were prepared and stained by haematoxylin and eosin (H\&E) and Masson trichrome stainings ${ }^{19}$. All 
sections were examined using light microscope. Sections were dewaxed in xylol, treated with graded alcohol and placed in water. The sections were stained in haematoxylin for 5-20 minutes, washed well in running tap water for 5 minutes. The excess stain was removed, and the slides were washed again. The slides were stained with $1 \%$ eosin for 10 minutes then washed in running tap water for 5 minutes, dehydrated through graded alcohols to xylol and mounted in a mixture of distyrene (a polystyrene), a plasticizer (tricresyl phosphate), and xylene (DPX). Then the slides were examined by expert in pathology. Sections were dewaxed in xylol, treated with graded alcohol and were placed in water. Sections were mordanted in Bounin 'solution, microwave for 1 minute, allowed to stand 15 minutes. Then they were washed for 5 minutes in running tap water to remove picric acid. Weigert 's working haematoxylin was done for 5 minutes, then, they were rinsed in distilled water. Biebrich scarlet for 5 minutes was done then they were rinsed in distilled water. Sections were put in phosphotungstic/phosphmolybdic acid for 10 minutes then the solution was discarded. They were transferred directly into Aniline blue for 5 minutes then rinsed in distilled water. They were put in $1 \%$ acetic acid for 1 minute, then solution was discarded. Then they were rinsed in distilled water, dehydrated through graded alcohol to xylol and mounted in DPX, clear Ed and coverslip. The slides were examined by expert in pathology and examined by image analysis. Olympus ${ }^{\circledR}$ digital camera was installed on Olympus ${ }^{\circledR}$ microscope with $1 / 2 \mathrm{X}$ photo adaptor, using $20 \mathrm{X}$ objective to photograph the slides. Intel ${ }^{\circledR}$ Core $\mathrm{I} 3{ }^{\circledR}$ was used. It is computer based with Video Test ${ }^{\circledR}$
Morphology® software (Russia) with a specific built-in routine for measurement of percentage area to analyze the images.

\subsection{Immunohistochemistry of osteocalcin}

Specimens were fixed in $4 \%$ buffered formaldehyde for 2 days at room temperature, and then decalcified in ethylenediaminetetraacetic acid (changed every 3 days) for 4 weeks. Decalcified tissues were then washed, dehydrated in gradient alcohol, embedded in paraffin wax, and cut into 4$\mu \mathrm{m}$-thick sections along the sagittal plane of the distal tibia. Immunohistochemical localization of osteocalcin was done using antibodies purchased from Sigma Company. Stained sections were examined qualitatively under light microscope with a digital camera.

\subsection{Statistical analysis}

Data were tabulated, coded then analyzed using the computer program SPSS (Statistical package for social science) version 23.0. Descriptive statistics were calculated in the form of mean \pm Standard deviation (SD). In the statistical comparison between the different groups, the significance of difference was tested using one-way ANOVA (analysis of variance) to compare between groups followed by post-hoc Tukey test. Also, Pearson correlation analyses were used. A minimum level of significance is considered if $\mathrm{p}$ is $<0.05$.

\section{Results}

\subsection{Exercise increases serum irisin level and} decreases serum carboxy-terminal cross-linked telopeptide of type I collagen (CTX) level in exercise -osteoporotic (EX+OST) group

Table (1) shows a significant increase in serum irisin level in exercise (EX) group as compared to control (C) group. While osteoporotic 
(OST) group showed a significant decrease in serum irisin level in relation to control (C) and exercise (EX) group. The exercise -osteoporotic $(\mathrm{EX}+\mathrm{OST})$ group had a significant increase in serum irisin level as compared to osteoporotic (OST) group. By comparing the serum carboxyterminal cross-linked telopeptide of type I collagen (CTX) level in different experimental groups, there was a significant decrease in CTX level in (EX) group as compared to $(\mathrm{C})$ group. On the other hand, there was a significant increase in (OST) group as compared to (C) and (EX) group. While there was a significant decrease in CTX level in exercise -osteoporotic $(\mathrm{EX}+\mathrm{OST})$ group relative to (OST) osteoporotic group. In addition, by comparing calcium level in different groups to the control (C) one, there were no significant differences.

Table (1): Comparison of the estimated biochemical parameters among the different experimental groups.

\begin{tabular}{|c|c|c|c|c|}
\hline$(\mathrm{n}=12)$ & $\begin{array}{c}\text { C } \\
\text { group }\end{array}$ & $\begin{array}{c}\text { EX } \\
\text { group }\end{array}$ & $\begin{array}{c}\text { OST } \\
\text { group }\end{array}$ & $\begin{array}{c}\text { OST+EX } \\
\text { group }\end{array}$ \\
\hline Irisin (ng\ml) & $65.55 \pm 2.99$ & $79.22 \pm 4.77$ & $25.46 \pm 3.26$ & $50.07 \pm 3.27$ \\
\hline P1 & & $<0.001 *$ & $<0.001 *$ & $<0.001^{*}$ \\
\hline $\mathbf{P 2}$ & & & $<0.001 *$ & $<0.001^{*}$ \\
\hline $\mathbf{P 3}$ & & & & $<0.001 *$ \\
\hline Calcium(mg $\backslash \mathrm{dl})$ & $9.55 \pm 0.14$ & $9.61 \pm 0.27$ & $9.34 \pm 0.42$ & $9.45 \pm 0.18$ \\
\hline P1 & & NS & NS & NS \\
\hline $\mathbf{P 2}$ & & & NS & NS \\
\hline $\mathbf{P 3}$ & & & & NS \\
\hline CTX (ng\ml) & $45.41 \pm 4.88$ & $33.06 \pm 3.71$ & $56.2 \pm 3.12$ & $49.77 \pm 3.18$ \\
\hline P1 & & $<0.001^{*}$ & $<0.001 *$ & $<0.05^{*}$ \\
\hline $\mathbf{P 2}$ & & & $<0.001 *$ & $<0.001 *$ \\
\hline P3 & & & & $<0.01 *$ \\
\hline
\end{tabular}

(C): control group. (EX): exercise group. (OST): osteoporotic group.

(EX+OST): exercise- osteoporotic group.

CTX I: carboxy-terminal cross-linked telopeptide of type I collagen

Test used: One-way ANOVA followed by post-hoc Tukey test P: Probability

*: significance $<0.05$

NS: non-significant

P1: significance as compared to control group

P2: significance as compared to exercise group

P3: significance as compared to Osteoporotic group 


\subsection{Results of dual-energy $x$-ray absorptiometry} (DEXA)

By comparing the parameters of bone measured by DEXA in different experimental groups, figures (1 A, B, E \& F) showed a significant changes in exercise (EX) group as compared to the control (C) group expressed as a significant increase in bone mineral content and bone mineral density. On the other hand, there were a significant decrease in both bone mineral content and density in osteoporotic (OST) group compared to both (EX) and (C) group (Figure $1 \mathrm{C}$, D, E \& F). Surprisingly, there was a significant increase in both mineral content and density in exercise osteoporotic (EX+ OST) group relative to (OST) group.

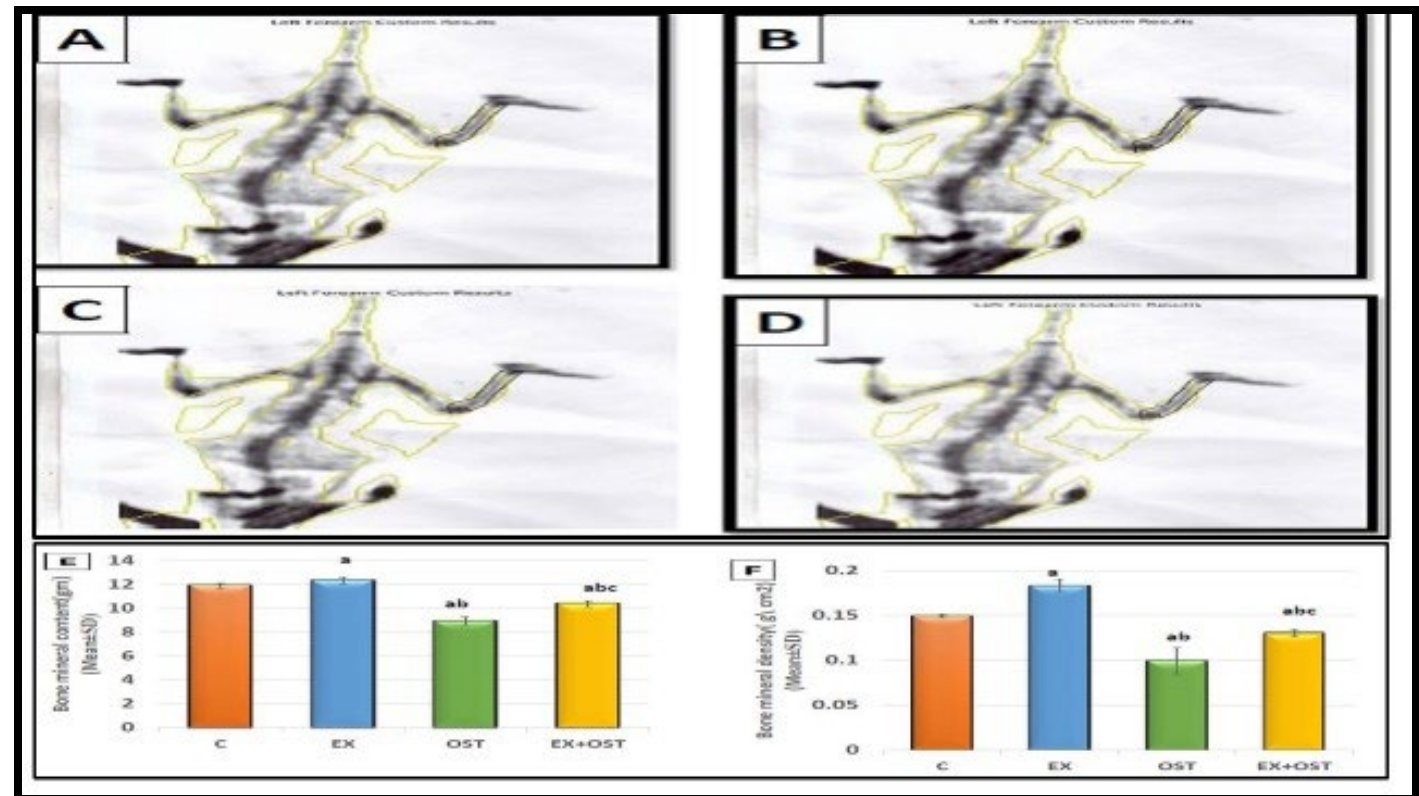

a: significance as compared to control (C) group.

$\mathrm{b}$ : significance as compared to exercise (EX) group.

c: significance as compared to osteoporotic (OST) group.

Test used: One-way ANOVA followed by post-hoc Tukey test

Figure (1): A) DEXA for control (C) group B) DEXA for exercise group (EX) C) DEXA for osteoporotic group (OST). D) DEXA for exercise- osteoporotic (EX+ OST) group. E) Bone mineral content in different experimental groups F) Bone mineral density in different experimental groups

\subsection{Cortical bone thickness and trabecular bone}

area \% increases relative to total bone area in

\section{exercise osteoporotic (OST+EX) group by}

\section{Hematoxylin and Eosin stain}

There is a normal bone architecture in both control (C) and exercise (EX) groups. Moreover, (EX) group showed a significant increase in cortical bone thickness and trabecular bone area \% relative to total bone area in comparison to control group. Nevertheless, there were a significant changes in tibial bone of osteoporotic (OST) group
(Figure 2). These changes were in the form of significant decrease in cortical bone thickness and trabecular bone area \% relative to total bone area as compared to all other groups. As regard exercise osteoporotic $(\mathrm{OST}+\mathrm{EX})$ group, there was a significant increase in both cortical bone thickness and trabecular bone area $\%$ relative to total bone area as compared to (OST) group (Figure 2). 


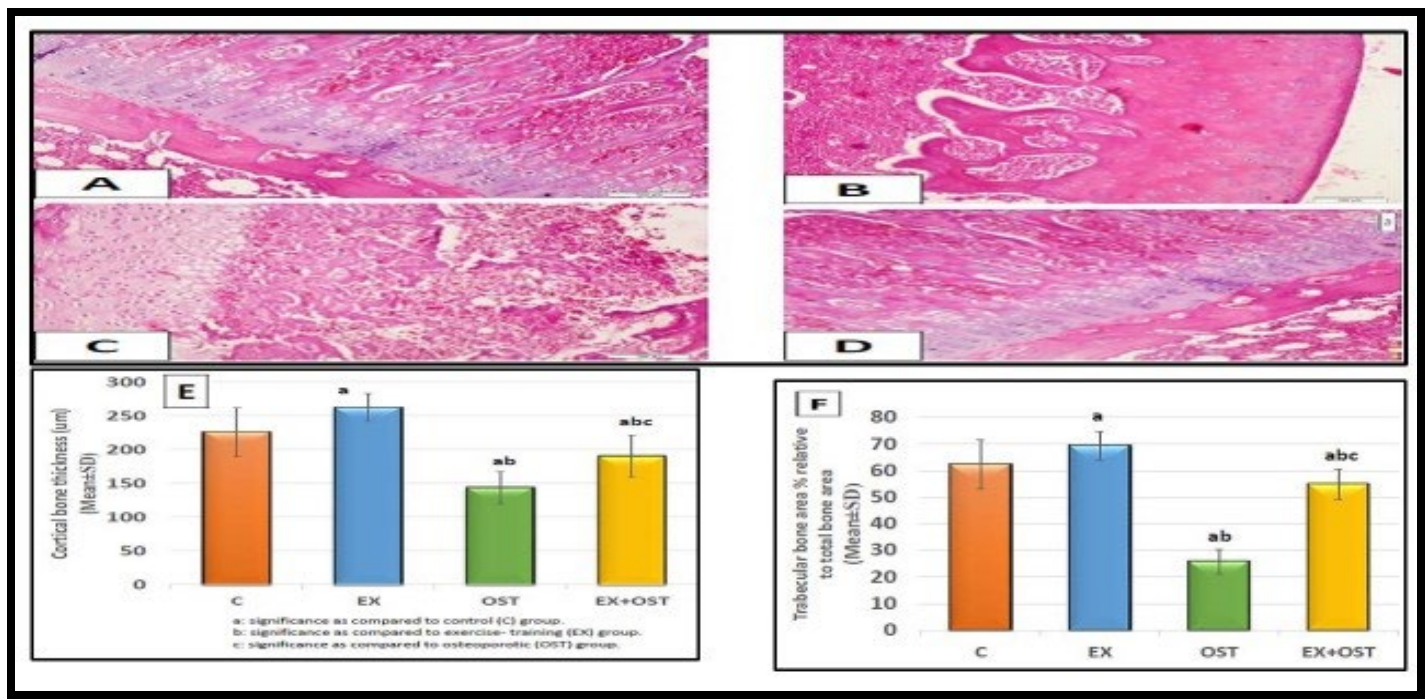

Test used: One-way ANOVA followed by post-hoc Tukey test

Figure (2): A) Bone section of control (C) group shows a complete bone trabeculae structure with ordered arrangement of trabeculae formed of outer lamellar and inner cancellous bone with network of anastomosing trabeculae enclosing bone marrow elements. (H\&E, 100X). B) Bone section of exercise (EX) group shows an exaggerated normal bone (H\&E, 100X) C) Bone section of osteoporotic (OST) group shows a reduction of bone trabeculae with observed thinning of the trabeculae with wide spacing between them (H\&E, 100X). D) Bone section of exercise - osteoporotic group shows a significant increase in both cortical bone thickness and trabecular bone area. (H\&E, 100X) E) Cortical bone thickness in different experimental groups F) Trabecular bone area \% relative to total bone area in different experimental groups.

\subsection{The percentage area stained with Masson's}

\section{Trichrome decreases in in exercise}

osteoporotic group (EX+OST)

Figure (3 C) shows diffuse marked significant increase in percentage area stained with Masson's Trichrome in osteoporotic (OST) group as compared to exercise (EX) and control (C) groups.
However, there was a significant decrease in percentage area stained with Masson's Trichrome in exercise osteoporotic group $(\mathrm{EX}+\mathrm{OST})$ relative to (OST) group (Figure 3 D). Surprisingly, by comparing the area stained with Masson's Trichrome in (EX) group and (C) group, there was no significant difference (Figure $3 \mathrm{E}$ ).
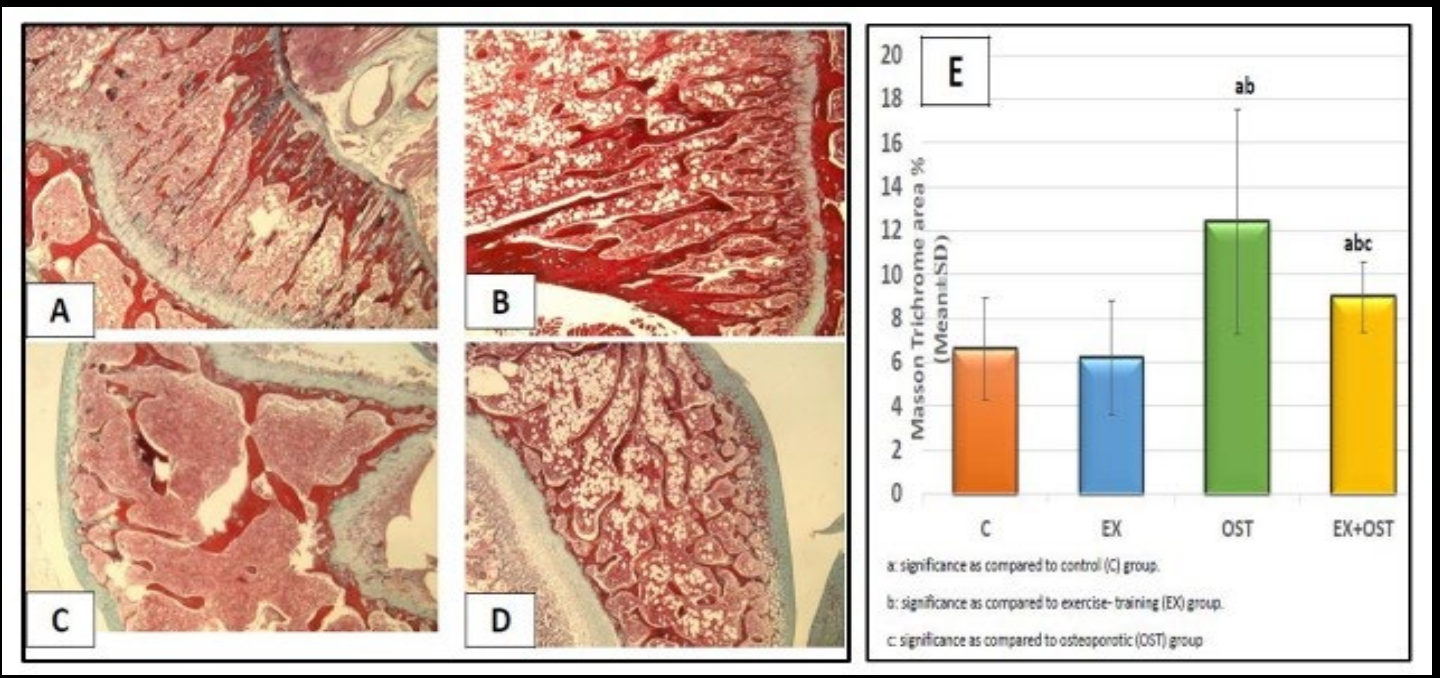

Test used: One-way ANOVA followed by post-hoc Tukey test

Figure (3): A) shows control (C) group (Masson's Trichrome X 400). B) shows exercise (EX) group (Masson's Trichrome X 400) C) shows osteoporotic (OST) group (Masson's Trichrome X 400). D) shows exercise -osteoporotic (EX+OST) group (Masson's Trichrome X 400). E) Comparison of percentage area of collagen deposition by Masson's Trichrome among the different experimental groups. 


\subsection{Analysis of osteocalcin (OCN)}

\section{immunohistochemistry examinations in}

different experimental groups

Figure (4) reveals a significant decrease in osteocalcin (OCN) activity in osteoporotic (OST) group as compared to both control (C) and exercise (EX) group. But, exercise- osteoporotic $(\mathrm{EX}+\mathrm{OST})$ group showed a significant increase in OCN activity relative to osteoporotic group (Figure 4 D). In addition, by comparing OCN activity in exercised group to the control group, there was no significant difference (Figure 4 B).
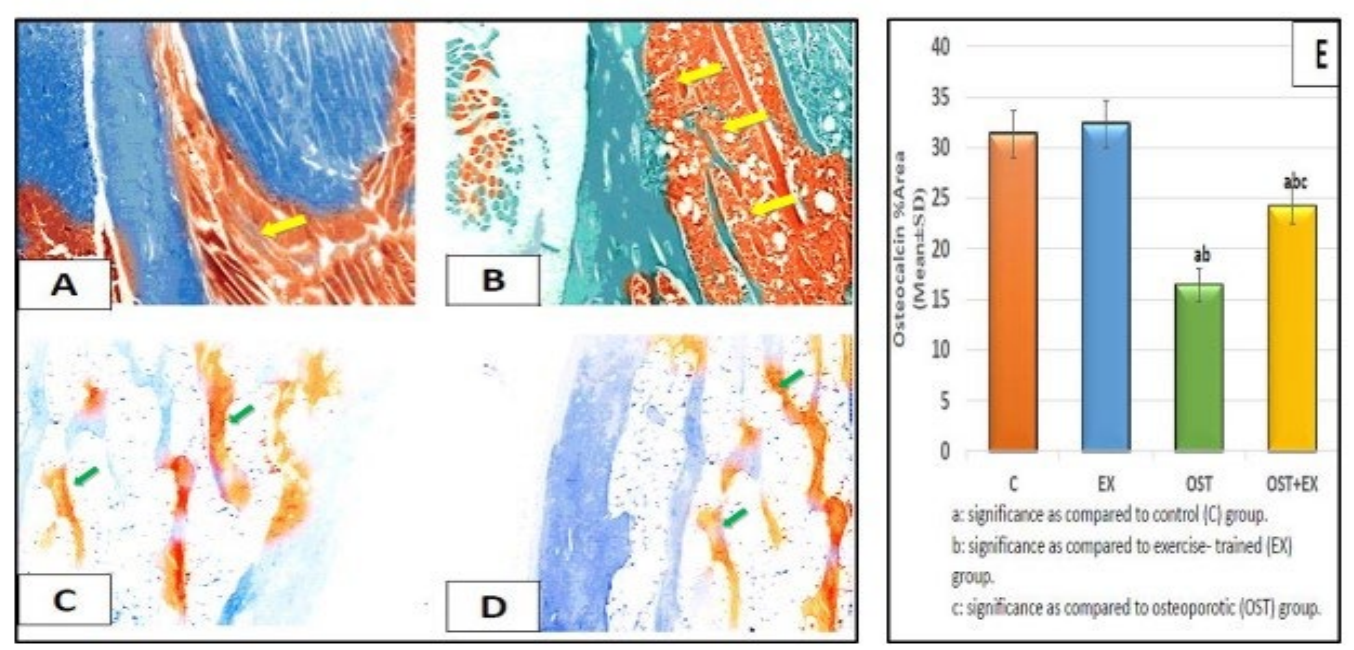

Test used: One-way ANOVA followed by post-hoc Tukey test

Figure (4): A) shows osteocalcin expression in control (C) group (X400). B) shows osteocalcin expression in exercise (EX) group (X400). C) shows osteocalcin expression in osteoporotic (OST) group (X 400). D) shows osteocalcin expression in exercise- osteoporotic (EX+OST) group (X 400). E) Comparison of osteocalcin activity among different experimental groups

\subsection{Correlation between serum irisin level and}

\section{the estimated parameters}

There is a significant positive correlation between serum irisin level and cortical bone thickness. Moreover, there is a significant positive correlation between serum irisin level and
Also, irisin shows a significant positive correlation with bone mineral content and density, osteocalcin activity and blood calcium level. However, serum irisin level shows a significant negative correlation with CTX and Masson's Trichrome percentage area (MTC \%A) (Table 3). trabecular bone area \% relative to total bone area.

Table (3): The correlation between serum irisin level and the estimated parameters:

\begin{tabular}{|l|l|l|}
\hline & \multicolumn{2}{|l|}{ Level of irisin $(\mathrm{ng} / \mathrm{ml})$} \\
\cline { 2 - 3 } & \multicolumn{1}{|c|}{$\mathrm{r}$} & \multicolumn{1}{c|}{$\mathrm{P}$} \\
\hline Cortical bone thickness um & 0.838 & $<0.001^{*}$ \\
\hline $\begin{array}{l}\text { Trabecular bone area \% relative to total bone } \\
\text { area }\end{array}$ & 0.903 & $<0.001^{*}$ \\
\hline MTC \%A & -0.02 & 0.894 \\
\hline Bone mineral density( g/ cm2) & 0.929 & $<0.001^{*}$ \\
\hline Bone mineral content(gm) & 0.957 & $<0.001^{*}$ \\
\hline Level of calcium: mg/dl & 0.353 & $<0.05$ * \\
\hline Osteocalcin \%A & 0.944 & $<0.001^{*}$ \\
\hline Serum CTX(ng/ml) & -0.852 & $<0.001^{*}$ \\
\hline
\end{tabular}

Pearson's correlation coefficient P: Probabilty *:significance $<0.05$ 


\section{Discussion}

Osteoporosis is the most common bone disease in humans, representing a major public health problem. It is caused by an altered balance between bone anabolism and catabolism (20). So, the aim of our work is to: (i) study the effect of exercise on irisin plasma level in rats (ii) identify the correlation between irisin level in blood and change in bone turnover in glucocorticoid induced osteoporosis.

In the present experiment, we studied, for the first time, the osteoprotective effects of endogenous irisin induced by exercise against osteoporotic changes produced by glucocorticoids (GC) treatment in a rat model. The assessment of bone metabolism includes two main aspects: bone mass and bone quality. The most common indicator of bone mass is bone mineral density (BMD) which is assessed by DEXA (21). It has been previously reported that a low BMD is one of the most important signs of osteoporosis (22). So, DEXA scan is the current gold standard test for the diagnosis of osteoporosis (23). By DEXA, the osteoporotic (OST) group showed significant decrease in bone mineral content and density. Moreover, it also showed deterioration of bone tissue and disturbance of bone architecture presented by histopathological examination and decreased osteocalcin expression by immunohistochemistry.

In the present study, the bone density of rats in the osteoporotic (OST) group was found to decline significantly compared to all other groups by DEXA. Glucocorticoids were found to depress Wnt signaling pathway through increasing the expression of Wnt-antagonists (24). This pathway normally promotes the differentiation of bone marrow stromal cells into mature osteoblasts, suppresses the apoptosis of osteoblasts, and enhances the proliferation and mineralization of osteoblasts (25).

Also, GCs increase the apoptosis of osteoblasts and mature osteocytes via activation of caspase 3 (26). Osteoblast/osteocyte apoptosis induced by GCs may involve decreased expression of the prosurvival factor B-cell lymphoma- extra large (BclXL) and increased expression of the proapoptotic factor BAK1 (BCL2 Antagonist/Killer 1) (27). Furthermore, GCs reduce osteoblast proliferation and differentiation, possibly as a result of GC-induced suppression of bone morphogenic protein-2 (BMP-2) and expression of core binding factor (28).

By H\&E, the osteoporotic group showed significant decrease in trabecular bone with observed thinning of the trabeculae with wide spacing between them. Histomorphometric studies have shown that GCs induce a thinning of the trabeculae and a loss of trabecular bone volume. Furthermore, disruption of cancellous bone microarchitecture (such as trabecular perforations) may occur, thus leading to an increase in the trabecular space and a decrease of the trabecular number (29).

Osteocalcin $(\mathrm{OCN})$ is the most abundant marker in the bone matrix, which is released by the osteoblasts to the outside of cells, reflecting the state of bone formation (30). OCN activity showed significant decrease in osteoporotic group. This agrees with previous studies which reported a diminished osteocalcin activity after glucocorticoids treatment (31). This occurs as glucocorticoids produce different metabolic 
phenotype in osteoblasts and reduce, but not totally abolish, their anabolic function and their ability to secrete OCN (32).

In the current study, the osteoporotic (OST) group showed a significant decrease in serum irisin level versus the control group. The decrease in serum irisin level may be attributed to osteoporosis that is associated with decrease in the expression of PPAR- $\gamma$ that leads to prevention of the interaction of PGC $1 \alpha$ with multiple transcription factors leading to decreased FNDC5 and irisin (33). Our current data confirm those by Anastasilakis et al. (34) who have shown lower serum irisin levels in postmenopausal women with previous osteoporotic fractures.

Our data did not reveal any significant changes in the serum calcium concentration in all groups as compared to control group. This result may be explained by the physiological regulatory mechanisms that control the calcium ion, and organized by calcitonin and parathyroid hormones. Since calcium participates in a variety of physiological processes and deviations in its serum level can affect the normal operations of the body (35). However, calcium level, herein, insignificantly decreased in (OST) group, which could be explained by the ability of glucocorticoids to reduce intestinal calcium absorption and increase renal calcium clearance (36).

The osteoporotic group showed a significant increase in CTX (carboxy-terminal cross-linked telopeptide of type I collagen) level. Detection of serum CTX-I content specifically reflects the resorption activity of osteoclasts. So, it is one of the most recommended investigation as specific bone resorption markers (37) and its serum level is positively correlated with bone resorption 38 . Increase in serum CTX-I in (OST) group might be due to increase bone and collagen degradation induced by glucocorticoids (39). This is confirmed by increase in percentage area stained with Masson's Trichrome in (OST) group as compared to all other groups.

In comparison to the control group, the exercise (EX) group showed significant increase in serum irisin level associated with a significant decrease in CTX-1 level.

Irisin levels increase during exercise training $(40,41)$. Similarly, other studies have showed a positive correlation between irisin and physical activity. There was no correlation between irisin levels and daily physical activity (39). Here, the significant increase in plasma irisin levels could be explained by effect of exercise which induces peroxisome proliferator-activated receptor gamma and peroxisome proliferator-activated receptor coactivator $1 \alpha(\mathrm{PGC}-1 \alpha)$ gene expression that promotes FNDC5 expression leading to splicing of FNDC5 to irisin $(42,43)$

In our study, the exercised group had significant increase in bone mineral density and content in the tibia when compared to any other group this might be due to the ability of exercise to preserve and promote greater bone formation. High frequency movement during swimming increases irisin level which increases BMD (44). Then irisin directly targets osteoblast and promotes osteoblast proliferation and bone mineralization via activating the p38 mitogen-activated protein kinase (p38 MAPK) and extracellular signal-regulated kinase (ERK) signaling pathways (45). This increases mRNA expression of osteoblast transcription regulators, including Runt-related 
transcription factor-2 (Runx2), a key transcription factor associated with osteoblast differentiation, and osterix (Osx, also know sp7). Runx2 acts by binding to osteoblast-specific element 2 (OSE2), which is found in the regulatory region of many osteoblast-specific genes (46). Increased expression of Runx 2 has been shown to be responsible for $\mathrm{p} 38$ in promoting osteogenesis. The p38 MAPK pathway is essential for skeletogenesis and bone homeostasis (47). ERK signaling pathway promoted osteoblast differentiation and bone mineralization48. Irisin also increases earlier osteoblast differentiation marker genes including osterix and bone sialoprotein (BSP) which play a vital role in matrix deposition and mineralization (49). This was evident, in the present study, by immunohistochemical staining that revealed increase in osteocalcin. Considering the above results, we conclude that irisin stimulates osteogenesis and help in bone mineralization. In young mice, r-irisin treatment increases cortical bone mineral density and positively modifies bone geometry, thus pro-osteoblastic genes are upregulated, and the activity of osteoblasts increases (50).

Here, the exercise (EX) group showed a significant increase in cortical bone thickness, trabecular bone density and trabecular bone area relative to total bone area. In this study, the increased mass of trabecular bone gained through swimming exercise was similar to that in other previous reports $(51,52)$. Also, our results are in agreement with others that indicated that irisin increases bone trabecular volume and cortical thickness in mice following intraperitoneal injection of irisin. These may be attributed to osteogenic effect of irisin. It directly stimulates new bone synthesis by osteoblasts as it enhances osteoblast differentiation through the Wnt- $\beta$ catenin signaling and inhibits osteoclast differentiation by suppressing the receptor activator of nuclear factor- $\mathrm{kB}$ ligand (RANK/RANKL) pathway. The osteogenic effect of irisin is also mediated initially by increasing activating transcription factor 4 (Atf4) mRNA expression, indicating enhanced precursor differentiation toward an osteoblast lineage (53).

Osteocalcin activity showed increase in exercised group. This might be due to increase in Runt related transcription factor 2 (Runx2) that is the specific transcription factor of osteoblasts and can play a leading role in regulating the expression of OCN genes (54).

By comparing to OST group, the $\mathrm{EX}+\mathrm{OST}$ group showed a significant increase in serum irisin level and significant decrease in CTX -1. This could be explained by the ability of exercise to induce irisin release from muscle which inhibits bone resorption, enhances bone formation and ameliorates bone loss $(55,56)$.

In the present study, a significant increase in bone mineral density and content were recorded in (EX+ OST) group versus OST group. The preceding exercise training in the $(\mathrm{EX}+\mathrm{OST})$ group might increase BMD. This could be explained by the ability of swimming to induce a substantial increase in calcium content of bone that compensate the osteoporotic effect of glucocorticoids (57).

Histological examination of Hematoxylin and Eosin stained bone sections showed that the exercise preceding induction of osteoporosis attenuated the deteriorated structure of the tibia in the exercise-trained osteoporotic $(\mathrm{EX}+\mathrm{OST})$ group 
which is observed by the increase in cortical thickness and trabecular number. This is contributed to the beneficial effects of muscle contraction and release of irisin on bone strength during exercise ${ }^{58}$.

In OST+EX group, Masson's Trichrome stained bone sections revealed a decrease in percentage area of collagen deposition, compared to osteoporotic group, as new bone formation replaces the area of degraded collagen deposition. There is, also, a significant increase of osteocalcin expression compared to OST group. This characterizes mature bones formation and a mineralization activity, which may be related to a satisfactory osseointegration process, featuring exercise effect on bone tissue ${ }^{59,60}$.

Finally, in this study, irisin released mainly by exercise, was found to be effectively improving different bone parameters such as bone mineral content and density. It is also positively correlated with cortical bone thickness and trabecular bone density. So, we can conclude that irisin is able to attenuate glucocorticoid- induced osteoporosis. These results indicate the therapeutic effects of irisin against glucocorticoids induced osteoporosis.

\section{Conclusions}

Irisin may be the link between the beneficial effect of exercise and the protection against osteoporosis. This conclusion is supported by the positive correlation between serum irisin and bone mineral content, bone mineral density, microstructure of the bone and osteocalcin expression percentage areas. It is also supported by the negative correlation between serum irisin and serum CTX. Based on these findings, recombinant irisin replacement therapy may be a crucial osteoprotective intervention for those patients whose plasma irisin level is reduced. Clinical trials may also be considered to validate the results in humans, following further pre-clinical studies.

\section{Acknowledgements}

The authors acknowledge Physiology Department and Medical Experimental Research Center MERC of Mansoura University for the significant contribution to the experimental part of the study.

Author contribution: All authors designed and performed research and wrote the paper.

Conflicts of Interest: The author declares no conflict of interest

\section{References}

1. WRIGHT, N. C., LOOKER, A. C., SAAG, K. G., CURTIS, J. R., DELZELL, E. S., RANDALL, S. \& DAWSON-HUGHES, B. 2014. The recent prevalence of osteoporosis and low bone mass in the United States based on bone mineral density at the femoral neck or lumbar spine. Journal of Bone and Mineral Research, 29, 2520-2526.

\section{FLORENCE, R., ALLEN, S., BENEDICT,} L., COMPO, R., JENSEN, A., KALOGEROPOULOU, D., KEARNS, A., LARSON, S., MALLEN, E. \& O'DAY, K. 2013. Diagnosis and treatment of osteoporosis. Bloomington (MN): Institute for Clinical Systems Improvement (ICSI). 
3. PAPAiOAnNOU, A., KENNEDY, C. C., IOANNIDIS, G., SAWKA, A., HOPMAN, W., PICKARD, L., BROWN, J., JOSSE, R., KAISER, S. \& ANASTASSIADES, T. 2009. The impact of incident fractures on health-related quality of life: 5 years of data from the Canadian Multicentre Osteoporosis Study. Osteoporosis International, 20, 703-714.

4. SANCHIS-GOMAR, F. \& PEREZ-QUILIS, C. 2014. The p38-PGC-1 $\alpha$-irisinbetatrophin axis: Exploring new pathways in insulin resistance. Adipocyte, 3, 67-68.

5. NELSON, H. D., HELFAND, M., WOOLF, S. H. \& ALLAN, J. D. 2002. Screening for postmenopausal osteoporosis: a review of the evidence for the US Preventive Services Task Force. Annals of internal medicine, 137, 529-541.

6. GOLOB, A. L. \& LAYA, M. B. 2015. Osteoporosis: screening, prevention, and management. Medical Clinics, 99, 587606.

7. MAUCK, K. F. \& CLARKE, B. L. 2006. Diagnosis, screening, prevention, and treatment of osteoporosis. Mayo Clinic Proceedings. Elsevier, 662-672.

8. CLARKE, B. L. \& KHOSLA, S. 2010. Physiology of bone loss. Radiologic Clinics, 48, 483-495.

9. COSMAN, F., DE BEUR, S. J., LEBOFF, M., LEWIECKI, E., TANNER, B., RANDALL, S. \& LINDSAY, R. 2014. Clinician's guide to prevention and treatment of osteoporosis. Osteoporosis international, 25, 2359-2381.

10. QIN, W., SUN, L., CAO, J., PENG, Y., COLLIER, L., WU, Y., CREASEY, G., LI, J., QIN, Y. \& JARVIS, J. 2013. The central nervous system (CNS)-independent anti-bone-resorptive activity of muscle contraction and the underlying molecular and cellular signatures. Journal of Biological Chemistry, 288, 13511-13521.

11. BOSTRöM, P., WU, J., JEDRYCHOWSKI, M. P., KORDE, A., YE, L., LO, J. C., RASBACH, K. A., BOSTRöM, E. A., CHOI, J. H. \& LONG, J. Z. 2012. A PGC1- $\alpha$-dependent myokine that drives brown-fat-like development of white fat and thermogenesis. Nature, 481, 463-468.

12. HOFMANN, T., ELBELT, U. \& STENGEL, A. 2014. Irisin as a muscle-derived hormone stimulating thermogenesis-a critical update. Peptides, 54, 89-100.

13. WU, Y.-C., CHEN, C.-H., MERCER, A. \& SOKOL, N. S. 2012b. Let-7-complex microRNAs regulate the temporal identity of Drosophila mushroom body neurons via chinmo. Developmental cell, 23, 202-209.

14. KURDIOVA, T., BALAZ, M., VICIAN, M., MADEROVA, D., VLCEK, M., VALKOVIC, L., SRBECKY, M., IMRICH, R., KYSELOVICOVA, O. \& BELAN, V. 2014. Effects of obesity, diabetes and exercise on Fndc5 gene expression and irisin release in human skeletal muscle and adipose tissue: in vivo 
and in vitro studies. The Journal of physiology, 592, 1091-1107.

15. HEE PARK, K., ZAICHENKO, L., BRINKOETTER, M., THAKKAR, B., SAHIN-EFE, A., JOUNG, K. E., TSOUKAS, M. A., GELADARI, E. V., HUH, J. Y. \& DINCER, F. 2013. Circulating irisin in relation to insulin resistance and the metabolic syndrome. The Journal of Clinical Endocrinology \& Metabolism, 98, 4899-4907.

16. LEMAIRE, K. K., THOMASSON, R., NOIREZ, P., JASPERS, R. T. \& VAN SOEST, A. 2017. Commentary: Validation of a ramp running protocol for determination of the true $\mathrm{VO} 2 \mathrm{max}$ in mice. Frontiers in physiology, 8, 330.

17. HENDERSON, G. C., FATTOR, J. A., HORNING, M. A., FAGHIHNIA, N., JOHNSON, M. L., MAU, T. L., LUKE-ZEITOUN, M. \& BROOKS, G. A. 2007. Lipolysis and fatty acid metabolism in men and women during the postexercise recovery period. The Journal of physiology, 584, 963-981.

18. BLACK, D. M., BILEZIKIAN, J. P., ENSRUD, K. E., GREENSPAN, S. L., PALERMO, L., HUE, T., LANG, T. F., MCGOWAN, J. A. \& ROSEN, C. J. 2005. One year of alendronate after one year of parathyroid hormone (1-84) for osteoporosis. New England Journal of Medicine, 353, 555-565.

19. PEDERSEN, B. K. \& FEBbRAIO, M. A. 2012. Muscles, exercise and obesity: skeletal muscle as a secretory organ. Nature Reviews Endocrinology, 8, 457465.

20. GANRY, O., BAUDOIN, C. \& FARDELLONE, P. 2000. Effect of alcohol intake on bone mineral density in elderly women: the EPIDOS study. American journal of epidemiology, 151, 773-780.

21. GARCES, M. F., PERALTA, J. J., RUIZLINARES, C. E., LOZANO, A. R., POVEDA, N. E., TORRES-SIERRA, A. L., ESLAVA-SCHMALBACH, J. H., AlZATE, J. P., SáNCHEZ, Á. Y. \& SANCHEZ, E. 2014. Irisin levels during pregnancy and changes associated with the development of preeclampsia. The Journal of Clinical Endocrinology \& Metabolism, 99, 2113-2119.

22. GAUdio, A., PENNISI, P., BRATENGEIER, C., TORRISI, V., LINDNER, B., MANGIAFICO, R. A., PULVIRENTI, I., HAWA, G., TRINGALI, G. \& FIORE, C. E. 2010. Increased sclerostin serum levels associated with bone formation and resorption markers in patients with immobilization-induced bone loss. The Journal of Clinical Endocrinology \& Metabolism, 95, 2248-2253.

23. GIMENO, R. E. \& MOLLER, D. E. 2014. FGF21-based pharmacotherapy-potential utility for metabolic disorders. Trends in Endocrinology \& Metabolism, 25, 303311. 
24. LIMA, F. D., STAMM, D. N., DELLAPACE, I. D., DOBRACHINSKI, F., DE CARVALHO, N. R., ROYES, L. F. F., SOARES, F. A., ROCHA, J. B., GONZáLEZ-GALLEGO, J. \& BRESCIANI， G. 2013. Swimming training induces liver mitochondrial adaptations to oxidative stress in rats submitted to repeated exhaustive swimming bouts. PloS one, 8, e55668.

25. LIN, C., JIANG, X., DAI, Z., GUO, X., WENG, T., WANG, J., LI, Y., FENG, G., GAO, X. \& HE, L. 2009. Sclerostin mediates bone response to mechanical unloading through antagonizing Wnt $/ \beta$-catenin signaling. Journal of bone and mineral research, 24, 1651-1661.

26. MAZZIOTTI, G., ANGELI, A., BILEZIKIAN, J. P., CANALIS, E. \& GIUSTINA, A. 2006. Glucocorticoidinduced osteoporosis: an update. Trends in Endocrinology \& Metabolism, 17, 144149.

27. MORENO-NAVARRETE, J. M., ORTEGA, F., SERRANO, M., GUERRA, E., PARDO, G., TINAHONES, F., RICART, W. \& FERNáNDEZ-REAL, J. M. 2013. Irisin is expressed and produced by human muscle and adipose tissue in association with obesity and insulin resistance. The Journal of Clinical Endocrinology \& Metabolism, 98, E769-E778.

28. MORSE, L., SUDHAKAR, S., LAZZARI, A., TUN, C., GARSHICK, E.,
ZAFONTE, R. \& BATTAGLINO, R. 2013. Sclerostin: a candidate biomarker of SCI-induced osteoporosis. Osteoporosis International, 24, 961-968.

29. MUNDY, G. R. \& ELEFTERIOU, F. 2006. Boning up on ephrin signaling. Cell, 126, 441-443.

30. NABI, G., AHMAD, N., ALI, S. \& AHMAD, S. 2015. Irisin: A Possibly New Therapeutic Target for Obesity and Diabetes Mellitus. World Journal of Zoology, 10, 205-210.

31. NAGAI, Y., GARRETT, K. P., OHTA, S., BAHRUN, U., KOURO, T., AKIRA, S., TAKATSU, K. \& KINCADE, P. W. 2006. Toll-like receptors on hematopoietic progenitor cells stimulate innate immune system replenishment. Immunity, 24, 801812.

32. PARK, K. H., ZAICHENKO, L., PETER, P., DAVIS, C. R., CROWELL, J. A. \& MANTZOROS, C. S. 2014. Diet quality is associated with circulating C-reactive protein but not irisin levels in humans. Metabolism, 63, 233-241.

33. PEDERSEN, L., IDORN, M., OLOFSSON, G. H., LAUENBORG, B., NOOKAEW, I., HANSEN, R. H., JOHANNESEN, H. H., BECKER, J. C., PEDERSEN, K. S. \& DETHLEFSEN, C. 2016. Voluntary running suppresses tumor growth through epinephrine-and IL-6-dependent NK cell mobilization and redistribution. Cell metabolism, 23, 554-562. 
34. ANASTASIllaKIS, A. D., POLYZOS, S. A., SARIDAKIS, Z.

G., KYNIGOPOULOS, G., SKOUVAKLIDOU, E. C., MOLYVAS, D., VASILOGLOU, M. F., APOSTOLOU, A., KARAGIOZOGLOU-LAMPOUDI, T. \& SIOPI, A. 2014. Circulating irisin in healthy, young individuals: day-night rhythm, effects of food intake and exercise, and associations with gender, physical activity, diet, and body composition. The Journal of Clinical Endocrinology \& Metabolism, 99, 32473255 .

35. RUAS, J. L., WHITE, J. P., RAO, R. R., KLEINER, S., BRANNAN, K. T., HARRISON, B. C., GREENE, N. P., WU, J., ESTALL, J. L. \& IRVING, B. A. 2012. A PGC-1 $\alpha$ isoform induced by resistance training regulates skeletal muscle hypertrophy. Cell, 151, 1319-1331.

36. SAMPSON, H. W., PERKS, N., CHAMPNEY, T. H. \& DEFEE, B. 1996. Alcohol consumption inhibits bone growth and development in young actively growing rats. Alcoholism: clinical and experimental research, 20, 1375-1384.

37. SELDIN, M. 2014. How muscle communicates nutritional status to regulate physiologic homeostasis. Johns Hopkins University.

38. TEITELBAUM, S. L. 2000. Bone resorption by osteoclasts. Science, 289, 1504-1508.
39. TEMUR, H. A., VARDAR, S. A., DEMIR, M., PALABıYıK, O., KARACA, A., GUKSU, Z., ORTANCA, A. \& SUT, N. 2015. The alteration of NTproCNP plasma levels following anaerobic exercise in physically active young men. Anatolian journal of cardiology, 15, 97.

40. THOMPSON, H. J., JIANG, W. \& ZHU, Z. 2009. Candidate mechanisms accounting for effects of physical activity on breast carcinogenesis. IUBMB life, 61, 895-901.

41. TIMMONS, J. A., WENNMALM, K., LARSSON, O., WALDEN, T. B., LASSMANN, T., PETROVIC, N., HAMILTON, D. L., GIMENO, R. E., WAHLESTEDT, C. \& BAAR, K. 2007. Myogenic gene expression signature establishes that brown and white adipocytes originate from distinct cell lineages. Proceedings of the National Academy of Sciences, 104, 4401-4406.

42. TOLOZA, F. J., MANTILLA-RIVAS, J. O., PEREZ-MATOS, M. C., RICARDOSILGADO, M. L., MORALESALVAREZ, M. C., PINZóN-CORTES, J. A., PEREZ-MAYORGA, M., AREVALO-GARCIA, M. L., TOLOSAGONZáLEZ, G. \& MENDIVIL, C. O. 2018. Plasma levels of myonectin but not myostatin or fibroblast-derived growth factor 21 are associated with insulin resistance in adult humans without diabetes mellitus. Frontiers in endocrinology, 9, 5 . 
41. TSUCHIYA, Y., ANDO, D., GOTO, K., KIUCHI, M., YAMAKITA, M. \& KOYAMA， K. 2014. High-intensity exercise causes greater irisin response compared with low-intensity exercise under similar energy consumption. The Tohoku journal of experimental medicine, 233, 135-140.

42. VESKOUKIS, A. S., KYPAROS, A., PASCHALIS, V. \& NIKOLAIDIS, M. G. 2018. A novel swimming performance test in rats. Chinese Journal of Physiology, 61, 144-151.

43. VICENTE-RODRíGUEZ, G. 2006. How does exercise affect bone development during growth? Sports Medicine, 36, 561569.

44. VILLARROYA, F. 2012. Irisin, turning up the heat. Cell metabolism, 15, 277-278.

45. VIRTANEN, J. K., MURSU, J., TUOMAINEN, T.-P. \& VOUTILAINEN, S. 2014. Dietary fatty acids and risk of coronary heart disease in men: the Kuopio Ischemic Heart Disease Risk Factor Study. Arteriosclerosis, thrombosis, and vascular biology, 34, 2679-2687.

46. VIRTANEN, K. A. 2014. BAT thermogenesis: linking shivering to exercise. Cell metabolism, 19, 352-354.

47. VOLPON, J. B., SILVA, A. V., FALCAI, M. J., LOUZADA, M. J. Q., ZAMARIOLI, 52. WARNER, S., SHEA, J., MILLER, S. \& SHAW, J. 2006. Adaptations in cortical
A., KOTAKE, B. G. D. S. \& ISSA, J. P. M. 2015. The osteogenic effects of swimming on bone mass, strength, and microarchitecture in rats with unloading-induced bone loss. Microscopy research and technique, 78, 784-791.

48. VOSS, M. W., VIVAR, C., KRAMER, A. F. \& VAN PRAAG, H. 2013. Bridging animal and human models of exerciseinduced brain plasticity. Trends in cognitive sciences, 17, 525-544.

49. WAHID, A., MANEK, N., NICHOLS, M., KELLY, P., FOSTER, C., WEBSTER, P., KAUR, A., FRIEDEMANN SMITH, C., WILKINS, E. \& RAYNER, M. 2016. Quantifying the association between physical activity and cardiovascular disease and diabetes: a systematic review and meta-analysis. Journal of the American Heart Association, 5, e002495.

50. WANG, S., MURA, C., SAWAYA, M. R., CASCIO, D. \& EISENBERG, D. 2002. Structure of a Nudix protein from Pyrobaculum aerophilum reveals a dimer with two intersubunit $\beta$-sheets. Acta Crystallographica Section D: Biological Crystallography, 58, 571-578.

51. WARMING, L., HASSAGER, C. \& CHRISTIANSEN, C. 2002. Changes in bone mineral density with age in men and women: a longitudinal study. Osteoporosis International, 13, 105-112.

and trabecular bone in response to mechanical loading with and without 
weight bearing. Calcified tissue international, 79, 395-403.

53. WATTS, J. J., ABIMANYI-OCHOM, J. \& SANDERS, K. M. 2013. Osteoporosis costing all Australian: a new burden of disease analysis-2012 to 2022 .

54. WRANN, C. D., WHITE, J. P., SALOGIANNNIS, J., LAZNIKBOGOSLAVSKI, D., WU, J., MA, D., LIN, J. D., GREENBERG, M. E. \& SPIEGELMAN, B. M. 2013. Exercise induces hippocampal BDNF through a PGC-1 $\alpha$ /FNDC5 pathway. Cell metabolism, 18, 649-659.

56. WU, J., BOSTRöM, P., SPARKS, L. M., YE, L., CHOI, J. H., GIANG, A.-H., KHANDEKAR, M., VIRTANEN, K. A., NUUTILA, P. \& SCHAART, G. 2012a. Beige adipocytes are a distinct type of thermogenic fat cell in mouse and human. Cell, 150, 366-376.

57. XU, B. 2013. BDNF (I) rising from exercise. Cell metabolism, 18, 612-614.

58. YANG, M., WEI, D., MO, C., ZHANG, J., WANG, X., HAN, X., WANG, Z. \&
XIAO, H. 2013. Saturated fatty acid palmitate-induced insulin resistance is accompanied with myotube loss and the impaired expression of health benefit myokine genes in $\mathrm{C} 2 \mathrm{C} 12$ myotubes. Lipids in health and disease, 12, 104.

59. YI, P., PARK, J.-S. \& MELTON, D. A. 2013. RETRACTED: Betatrophin: A Hormone that Controls Pancreatic $\beta$ Cell Proliferation. Elsevier.

60. ZACARIAS, A. C., BARBOSA, M. A., GUERRA-Sá, R., DE CASTRO, U. G. M., BEZERRA, F. S., DE LIMA, W. G., CARDOSO, L. M., SANTOS, R. A. S. D., CAMPAGNOLE-SANTOS, M. J. \& ALZAMORA, A. C. 2017. Swimming training induces liver adaptations to oxidative stress and insulin sensitivity in rats submitted to high-fat diet. Redox Report, 22, 515-523. 